\title{
Implementación de la evaluación formativa por estudiantes de Pedagogía en Educación Física \\ Implementation of the formative evaluation by students of Pedagogy in Physical Education
}

\author{
*María Vergara Tapia, *Mónica Flores Bastias, **Lucía Illanes Aguilar, ***Felipe Poblete-Valderrama \\ *Universidad Mayor (Chile), **Universidad Andrés Bello (Chile), ***Universidad Católica de la Santísima Concepción (Chile)
}

Resumen: La presente investigación se centra en el ámbito de la Formación Inicial Docente (FID) en Pedagogía en Educación Física (PEF). El objetivo es conocer la forma en que estudiantes en proceso de FID en PEF implementan la evaluación formativa en las clases que realizan en su práctica profesional. Se lleva a cabo mediante un enfoque cuantitativo, descriptivo, no experimental transversal, en donde se consideraron a 64 estudiantes de una institución de educación superior privada de Chile. El instrumento de recolección de información que se utilizó fue un guión de observación aplicado por parte de profesores supervisores de práctica profesional el cual considera las dimensiones de: a) Retroalimentación, b) Elaboración e implementación y c) Respuesta de los y las escolares. Los resultados verifican la aplicación de retroalimentación, apoyo diferenciado según lo requieren los y las escolares y tratamiento del error; pero no aplican en forma sistemática la autoevaluación ni la coevaluación, ni registro de lo realizado por los estudiantes en clases. Se puede concluir que si bien se incorpora la evaluación formativa en el desarrollo de la práctica profesional por parte de estudiantes en proceso de FID en PEF, estos no propician espacios de diálogo respecto al proceso evaluativo (autoevaluación y evaluación por pares).

Palabras Claves: Evaluación Formativa, Formación de Profesores, Práctica Profesional, Pedagogía en Educación Física, Educación Superior.

\begin{abstract}
This research focuses on the field of Initial Teacher Training (FID) in Pedagogy in Physical Education (PEF). The objective is to know the way in which students in the FID process at PEF implement the formative assessment in the classes they take in their professional practice. It is carried out through a quantitative, descriptive, non-experimental cross-sectional approach, where 64 students from a private higher education institution in Chile were considered. The information collection instrument that was used was an observation script applied by supervising professors of professional practice which considers the dimensions of: a) Feedback, b) Preparation and implementation and c) Response of the schoolchildren. The results verify the application of feedback, differentiated support as required by the schoolchildren and treatment of the error; but they do not apply in a systematic way self-evaluation or coevaluation, nor record of what the students have done in class. It can be concluded that although formative evaluation is incorporated in the development of professional practice by students in the FID process in PEF, they do not provide spaces for dialogue regarding the evaluation process (self-evaluation and peer evaluation).
\end{abstract}

Key Words: Formative Assessment, TeacherTraining, Professional Practice, Pedagogy in Physical Education, Higher Education.

\section{Introducción}

Conocer de qué forma los(as) estudiantes en proceso de Formación Inicial Docente (FID) en Pedagogía en Educación Física (PEF) implementan la evaluación formativa en las clases que realizan en su práctica profesional ha tomado un renovado interés en procesos de investigación educativa en el ámbito de la evaluación. Ya que se busca conocer el uso de la evaluación como una actividad crítica de aprendizaje, en el sentido que por ella se adquiere conocimiento (Álvarez Méndez, 1993) y como se desarrolla específicamente en las clases que

Fecha recepción: 18-12-22. Fecha de aceptación: 13-09-21

Felipe Poblete-Valderrama

felipepobletev@gmail.com llevan a cabo los(as) estudiantes en práctica profesional (final) en la carrera de Pedagogía en Educación Física.

La Educación Superior se encuentra ante la necesidad de avanzar desde un paradigma «centrado en el profesor» hacia un paradigma «centrado en el estudiante», de ahí la obligación de desarrollar una enseñanza basada en las competencias mediante la aplicación de metodologías activas y, de manera conjunta, emplear modelos alternativos de evaluación (López-Pastor et al., 2020). La evaluación en la FID en PEF debe avanzar con el objetivo de acrecentar la competencia en evaluación de los futuros profesores de Educación Física (Dinan Thompson \& Penney, 2015). Por ello es importante verificar lo que evidencian respecto al manejo de la evaluación formativa el profesorado en proceso de FID en PEF ya que busca generar procesos de mejora y apren- 
dizaje en tres sentidos: (a) optimizar los procesos de aprendizaje y producciones del alumnado; (b) perfeccionar la práctica docente; y, (c) reconducir los procesos de enseñanza-aprendizaje que se llevan a cabo en el aula (López Pastor et al., 2020). Este proceso de mejora y optimización se realiza desde una perspectiva humanizadora, sin una finalidad calificadora, (López-Pastor, 2009; López-Pastor \& Pérez-Pueyo, 2017) y la utilización correcta del feedback es un punto clave.

El enfoque de evaluación para el aprendizaje surge como un «intento consciente de hacer de la evaluación un elemento productivo en el aprendizaje, mejorando las interacciones entre docente y estudiantes y entre estudiantes» (Stobart, 2006, p.169), que valora las dimensiones contextuales, la diversificación de estrategias de evaluación y la retroalimentación para la autorregulación y autonomía de los estudiantes.

Una evaluación formativa debe emplearse para ajustar las prácticas pedagógicas, una prueba no es la única fuente de información que puede considerarse como evaluación formativa, también es posible emplear observaciones, el trabajo oral, así como también, los errores de los alumnos (Agencia de la Calidad de la Educación, 2016).

Esta evaluación formativa se enmarca en los postulados de la teoría constructivista del aprendizaje, donde el estudiante tiene un rol preponderante respecto a la construcción de su aprendizaje. Por otro lado la evaluación con base en el proceso se caracteriza por permitir mejorar la enseñanza aprendizaje a través de la retroalimentación y detectar a tiempo las causas del problema e identificar sus fallas y errores. Por lo tanto, la evaluación formativa, considera la construcción de aprendizajes en el proceso mismo de la enseñanza, teniendo como referencia la evaluación, que implica en la práctica, el evaluar para aprender.

(Black \& William, 2009) resaltan la importancia de utilizar la información que se genera en «el hacer» en la clase: «la práctica en una clase es formativa en la medida en que la evidencia acerca de los logros de los estudiantes es obtenida, interpretada y usada por docentes, aprendices o sus pares». Al respecto, la co-evaluación tiene muchas ventajas: promueve la cohesión en el grupo, incrementa la motivación de los estudiantes, facilita darles devoluciones y en el caso de la autoevaluación que implica motivación del estudiante para aprender, es necesario estimularla para que comprenda lo que está aprendiendo y pueda desarrollar procesos de metacognición que le permitan monitorear su propia forma de aprender (Ravela, Picaroni \& Loureiro. 2017).
Esto rescata la necesidad de hacer un uso sistemático de la información, lo que aporta para una toma de decisiones pertinente, porque tiene una base en las evidencias obtenidas en el proceso de enseñanza aprendizaje.

Cuando no se utiliza la evaluación formativa, de acuerdo con (Ramírez, 2011), se priva al estudiante la oportunidad de cometer errores y corregirlos, este trata de ocultar sus deficiencias y no asume la responsabilidad de evaluarse a sí mismo y de adquirir conciencia de cómo está aprendiendo y lo que debe hacer para mejorar.

Los procesos de revisión colectiva de trabajos y desempeños ponen en juego habilidades cognitivas y socioemocionales que amplían el campo de acción mental de todos los involucrados.

Por lo que se establecen preguntas claves en el uso de evaluación formativa para el estudiante: ¿a dónde tratas de ir? ¿dónde estás ahora? ¿cómo puedes llegar ahí? (William \& Thompson, 2007). Con la intención de que el estudiante conozca los objetivos de aprendizaje que va a aprender y los criterios con los que va a ser evaluado, se torna fundamental para que se sitúe en lo que tiene que hacer para lograr los aprendizajes que debe conseguir.

El profesorado debe tener claro los conocimientos previos que requiere el estudiante en proceso de FID en PEF y que facilitarán la formación, definir tareas y actividades que permitan al estudiante alcanzar estos aprendizajes será clave. Además, aplicar la retroalimentación, que se genera en los resultados de aprendizaje que evidencia el estudiante en el proceso y a término de este. En el contexto universitario centran su acción en aprendizajes orientados a la práctica profesional con un marcado protagonismo del alumnado en su propio proceso formativo (López, Benedito \& León, 2016).

La retroalimentación es especialmente eficaz cuando dirige su atención a cualidades particulares del trabajo del estudiante, en relación con criterios establecidos y a la vez, proporciona a este una guía sobre qué hacer para mejorar.

Ramírez (2011) afirma que la evaluación formativa es la más importante y viable para mejorar la calidad educativa en general y en todos los niveles, porque permite localizar las deficiencias para remediarlas, utilizando parámetros de aprendizaje, que finalmente le permitan al estudiante resolver los problemas que dificultan su progreso, para ello, esta debe realizarse de manera frecuente y sistemática; también, se dirige a detectar aquellas zonas en las que, en el caso del desempeño docente, el profesor necesita reforzar para mejo- 
rar su labor en el aula.

La eficacia de la retroalimentación aumenta cuando se plantean las siguientes preguntas: ¿cuál es el error principal? ¿cuál es la razón probable de que el estudiante cometa este error? ¿cómo puedo guiar al estudiante para que evite el error en un futuro? (Ramírez, 2011).

$\mathrm{Al}$ respecto (Broadfoot, et al., 1999), centran el foco de la evaluación para el aprendizaje en cómo esta involucra a los estudiantes: proveer retroalimentación efectiva a los alumnos, involucrarlos en su propio aprendizaje, ajustar la enseñanza considerando los resultados de la evaluación, reconocer la influencia que tiene la evaluación sobre la motivación y la autoestima de los alumnos, las que son esenciales en el aprendizaje y la necesidad que los alumnos sean capaces de evaluarse y comprender cómo mejorar.

Para efectos de este estudio, se mantiene el término de evaluación formativa, por ser el que manejan habitualmente los y las profesores/as en nuestra realidad educativa nacional. La evaluación formativa de los aprendizajes no es un tema nuevo (Hamodi, López \& López, 2015), pero sí es controvertido, en el sentido que se reconoce la importancia de aplicar esta evaluación que responde a la intención de intervenir y mejorar los procesos educativos, pero que se considera complicado, difícil de llevar a cabo, que requiere de tiempo y esfuerzos que no siempre el profesor puede o se le dificulta asumir y de lo cual hay pocos estudios en nuestro contexto educativo nacional (Agencia de la Calidad de la Educación, 2016). Por lo mismo, resulta necesario considerar las normativas que se han implementado y que plantean una consideración clara de aplicar evaluación formativa en los procesos de enseñanza aprendizaje en el ámbito escolar (Decreto Supremo N67, 2016). Para ello, tiene sentido el aporte que hace (Asún, et al., 2020) que plantea potenciar el buen uso de la retroalimentación, para reflexionar y promover competencias cognitivas de orden superior en los aprendizajes de los futuros docentes, con objeto de poder ampliar y mejorar esta competencia en la formación inicial y permanente.

Si nos situamos en el contexto de la FID en PEF, y en el concepto de evaluación formativa, surgen preguntas al respecto: ¿cuál es el sentido con que se evalúa en FID en PEF? ¿los y las estudiantes de Educación Física en FID conocen las implicancias de la evaluación formativa en el proceso de enseñanza aprendizaje que tienen los y las escolares? ¿conocen procedimientos que les permitan aplicar evaluación formativa? ¿aplican evaluación formativa en sus clases? Algunas de estas interrogantes orientarán el desarrollo de este estudio, en especial, aquella que busca verificar el uso y las formas en que se aplica evaluación formativa en las clases por estos profesores en FID en PEF y la respuesta de los y las escolares respecto de estas intervenciones.

\section{Material y Método}

El presente estudio se desarrolla mediante un enfoque cuantitativo, con un alcance descriptivo y un diseño no-experimental transversal. Con el objetivo de conocer la implementación de la evaluación formativa por parte de profesores en FID en PEF en su práctica profesional pertenecientes a una institución de educación superior chilena.

\section{Población y Muestra:}

La muestra fue conformada por 64 estudiantes (49 de sexo masculino y 15 de sexo femenino) en proceso de FID en PEF de una institución de educación superior privada de Chile que realizaron su práctica profesional en 55 establecimientos educacionales (40 particulares, 6 particulares subvencionados, 9 municipales).

\section{Instrumento de evaluación}

El instrumento es un guión de observación que establece un apartado para recabar datos sociodemográficos. Además, considera tres dimensiones: a) Dimensión Retroalimentación, b) Dimensión Elaboración e implementación, c) Respuesta de los escolares.

Para la construcción y validación del instrumento se ejecutaron tres etapas, la primera etapa consistió en la elaboración del instrumento por parte de los investigadores responsables (se definió las dimensiones y enunciados de cada una), en una segunda etapa el instrumento se envió a dos expertos para su validación y retroalimentación externa (lingüista y técnica), en una tercera etapa se ajustó y aplicó el instrumento final a una muestra reducida de estudiantes (10 estudiantes) prueba piloto para su último ajuste antes de aplicación oficial, se consideró un procedimiento de confiabilidad inter observador con un valor de 0.85 con el objetivo de tener la versión definitiva del instrumento validada. Las observaciones fueron registradas mediante una escala que considera la presencia o ausencia de los indicadores Correspondientes a cada una de las dimensiones precedentemente señaladas.

Los observadores que aplicaron la pauta son profesores que cumplen la función de supervisión en la práctica profesional de los estudiantes de FID en PEF en 
centros educacionales de dependencia municipal, particular y particular subvencionados de Chile. La preparación de los observadores se realizó a través de una jornada de capacitación a cargo de las investigadoras donde se revisó el modelo de evaluación para el aprendizaje, se trabajó con el guión de observación para clarificar y aunar criterios. Cada profesor supervisor tiene a su cargo entre cinco y diez estudiantes, y para efectos de este estudio, realizaron tres observaciones en promedio.

Para el análisis de los datos se sumaron todos los ítems correspondientes a las distintas dimensiones. Se determinó la frecuencia en que se manifestó cada indicador y se determinó el porcentaje en el que se presenta, respecto a «si lo hace», «no lo hace»y «limitado de hacer».

\begin{tabular}{|c|c|c|}
\hline Dimensiones & Acciones del Profesor en Formación (FID en PEF) & \begin{tabular}{|l|l|l|l}
1 & 23 \\
\end{tabular} \\
\hline \multirow{10}{*}{ Retroalimentació } & Observa y señala cómo mejorar. & \\
\hline & Hace preguntas claves. & \\
\hline & Indicaciones precisas y claras. & \\
\hline & Retroalimenta respecto a los resultados obtenidos en las tareas. & \\
\hline & Refuerza logros al cierre de la clase y señala tareas. & \\
\hline & Dedica más tiempo a los estudiantes con más dificultades. & \\
\hline & Observa y estimula a los estudiantes con mayores problemas para & \\
\hline & logra aprendizajes. & \\
\hline & Solo señala que la tarea está mal hecha, pero no como mejorarla. & \\
\hline & $\begin{array}{l}\text { Da retroalimentación en forma positiva, sin descalificar a quien tiene } \\
\text { dificultades. }\end{array}$ & \\
\hline \multirow{8}{*}{$\begin{array}{l}\text { Elaboración e } \\
\text { implementación }\end{array}$} & Incentiva que los estudiantes se apoyen evaluándose entre sí. & \\
\hline & Genera acuerdos en relación a los criterios de evaluación y propicia & \\
\hline & la participación durante la autoevaluación y coevaluación. & \\
\hline & Socializa los resultados, incorpora la coevaluación y autoevaluación & \\
\hline & en el proceso de evaluación. & \\
\hline & Evaluación sumativa, usa un instrumento que evalúa aprendizajes & \\
\hline & planteados en los objetivos y que son conocidos por los y las & \\
\hline & $\begin{array}{l}\text { estudiantes. } \\
\text { El instrumento aplicado establece dimensiones e indicadores. }\end{array}$ & \\
\hline \multirow{6}{*}{$\begin{array}{l}\text { Respuesta de los } \\
\text { escolares }\end{array}$} & Cuando recibe correcciones o aportes las incorpora en sus siguientes & \\
\hline & ejecuciones, tareas o actividades. & \\
\hline & Solicita al profesor que lo observe y le diga cómo está su ejecución. & \\
\hline & Se autoevalúa y comparte con el profesor sus juicios valorativos. & \\
\hline & Participa en la evaluación de pares en forma colaborativa & \\
\hline & considerando criterios negociados previamente. & \\
\hline
\end{tabular}

\section{Resultados}

A continuación, se procede a presentar los resultados de la presente investigación.

La tabla 1 registro de acciones dimensión retroalimentación expone los porcentajes de estudiantes de FID en PEF que durante el desarrollo de las sesiones de práctica profesional aplican acciones de la evaluación formativa, los resultados permiten señalar que los indicadores como «observa y señala cómo mejorar» y «da retroalimentación en forma positiva, sin descalificar a quien tiene dificultades», evidencian intervenciones para apoyar los aprendizajes, pese a ello hay 22 clases $(13,9 \%)$ en donde no ocurre esto. Respecto al indicador «solo señala que la tarea está mal hecha, pero no cómo mejorarla», se muestra un comportamiento adecuado a lo requerido al uso de evaluación formativa. Esto demuestra que en la mayoría de las clases se hace retroalimentación correctiva a los escolares.
La tabla 2 cantidad de estudiantes en FID en PEF en aplicación de indicadores dimensión retroalimentación presenta las frecuencias en las cuales se puede apreciar que 30 estudiantes en FID en PEF aplican entre 7 a 8 indicadores durante la evaluación formativa en el desarrollo de su práctica profesional.

La tabla 3 registro de acciones dimensión elaboración e implementación muestra los porcentajes de estudiantes de FID en PEF que durante el desarrollo de las sesiones de práctica profesional aplican acciones de la evaluación formativa, los resultados permiten señalar que en el indicador «Incentiva que los estudiantes se apoyen evaluándose entre sí», es bajo el porcentaje $(32,3 \%)$ de las clases en que se observa la realización esta acción, al igual que en el indicador «Genera acuerdos en relación con los criterios de evaluación y propicia la participación durante la autoevaluación y coevaluación» presentando solo un $(19,6 \%)$ en donde se observa que si se realiza la acción. Lo que expone que

\begin{tabular}{|c|c|c|c|}
\hline \multicolumn{2}{|l|}{ Registro de acciones dimensión retroalimentación, porcentaje. } & & \\
\hline Acciones del Profesor en Formación (FID en PEF) & Si lo & No lo 1 & Limitado \\
\hline Observa y señala cómo mejorar. & 96,8 & 3,2 & 0 \\
\hline Hace preguntas claves. & 81 & 19 & 0 \\
\hline Indicaciones precisas y claras. & 89,2 & 10,8 & 0 \\
\hline Retroalimenta respecto a los resultados obtenidos en las tareas. & 50,6 & 46,8 & 2,6 \\
\hline Refuerza logros al cierre de la clase y señala tareas. & 74 & 23,4 & 2,6 \\
\hline Dedica más tiempo a los estudiantes con más dificultades. & 68,9 & 31 & 0 \\
\hline $\begin{array}{l}\text { Observa y estimula a los estudiantes con mayores problemas para logra } \\
\text { aprendizajes. }\end{array}$ & 82,2 & $17,8 \%$ & 0 \\
\hline Solo señala que la tarea está mal hecha, pero no como mejorarla. & 9,4 & $90,6 \%$ & 0 \\
\hline $\begin{array}{l}\text { Da retroalimentación en forma positiva, sin descalificar a quien tiene } \\
\text { dificultades. }\end{array}$ & 86 & $13,9 \%$ & 0 \\
\hline \multicolumn{4}{|l|}{ Tabla 2} \\
\hline \multicolumn{4}{|c|}{ Cantidad de estudiantes en FID en PEF en aplicación de indicadores dimensión retroalimentación., } \\
\hline \multicolumn{4}{|c|}{$\mathrm{N}^{\circ}$ de estudiantes de FID en PEF que Si lo hace } \\
\hline \multicolumn{4}{|l|}{8} \\
\hline \multicolumn{4}{|l|}{19} \\
\hline \multicolumn{4}{|l|}{16} \\
\hline \multicolumn{4}{|l|}{11} \\
\hline \multicolumn{4}{|l|}{4} \\
\hline \multicolumn{4}{|l|}{2} \\
\hline \multicolumn{4}{|l|}{ Tabla 3} \\
\hline \multicolumn{4}{|l|}{ Registro de acciones dimensión Elaboración e implementación, porcentaje. } \\
\hline Acciones del Profesor en Formación (FID en PEF) & $\begin{array}{l}\text { Si lo } \\
\text { hace }\end{array}$ & $\begin{array}{l}\text { No lo } 1 \\
\text { hace }\end{array}$ & $\begin{array}{c}\text { Limitado } \\
\text { hacer }\end{array}$ \\
\hline \multirow{2}{*}{$\begin{array}{l}\text { Incentiva que los estudiantes se apoyen evaluándose entre sí. } \\
\text { Genera acuerdos en relación a los criterios de evaluación y propicia la } \\
\text { participación durante la autoevaluación y coevaluación. }\end{array}$} & 32,3 & 66,4 & 1,3 \\
\hline & 19,6 & 79,2 & 1,2 \\
\hline $\begin{array}{l}\text { Socializa los resultados, incorpora la coevaluación y autoevaluación en el } \\
\text { proceso de evaluación. }\end{array}$ & 21 & 74 & 5 \\
\hline \multirow{2}{*}{$\begin{array}{l}\text { Evaluación sumativa, usa un instrumento que evalúa aprendizajes } \\
\text { planteados en los objetivos y que son conocidos por los y las estudiantes. } \\
\text { El instrumento aplicado establece dimensiones e indicadores. }\end{array}$} & 37 & 54 & 9 \\
\hline & 36 & 58 & 6 \\
\hline Tabla 4 & & & \\
\hline $\begin{array}{l}\text { Cantidad de estudiantes en FID en PEF en aplicación de indicadores dimensión ela } \\
\text { Cantidad Indicadores Aplicados } \\
\mathrm{N}^{\circ} \text { de estudiantes de FID e }\end{array}$ & $\begin{array}{ll}\text { aboración } \\
\text { en PEF }\end{array}$ & $\frac{n \text { e implem }}{\text { que Si lo }}$ & $\frac{\text { mentación. }}{\text { o hace }}$ \\
\hline 5 & & & \\
\hline 4 & & & \\
\hline 3 & & & \\
\hline 24 & & & \\
\hline 14 & & & \\
\hline 16 & & & \\
\hline Tabla 5 & & & \\
\hline Respuestas de los escolares a la intervención del estudiante FID en PEF en evaluaci & ión form & & \\
\hline Indicadores & & $\begin{array}{l}\text { Silo } \\
\text { hace }\end{array}$ & $\begin{array}{c}\text { No lo } \\
\text { hace }\end{array}$ \\
\hline $\begin{array}{l}\text { Cuando recibe correcciones o aportes las incorpora en sus sig } \\
\text { ejecuciones, tareas o actividades. }\end{array}$ & guientes & 121 & 40 \\
\hline Solicita al profesor que lo observe y le diga cómo está su ejecución. & & 85 & 76 \\
\hline Se autoevalúa y comparte con el profesor sus juicios valorativos. & & 58 & 103 \\
\hline $\begin{array}{l}\text { Participa en la evaluación de pares en forma colaborativa considerando cr } \\
\text { negociados previamente. }\end{array}$ & iterios & 22 & 139 \\
\hline
\end{tabular}


estas acciones, que claramente tienen que ver con el uso de evaluación formativa, no están presentes en la mayoría de las sesiones de clases observadas.

La tabla 4 Cantidad de estudiantes en FID en PEF en aplicación de indicadores dimensión elaboración e implementación se puede apreciar que en la dimensión que considera los elementos propios y más característicos de la evaluación formativa, como la participación durante la autoevaluación y coevaluación, establecer criterios en forma conjunta, conocer previamente los instrumentos, socializar los resultados, no son aplicados por los profesores en FID en las sesiones observadas, ya que solo dos estudiantes aplican 4 de estos indicadores y la mayoría (24 estudiantes de FID en PEF) aplica solo 2 de ellos.

La tabla 5 Respuestas de los escolares a la intervención del estudiante FID en PEF en evaluación formativa señala que respecto al indicador «Cuando recibe correcciones o aportes las incorpora en sus siguientes ejecuciones, tareas o actividades», 121 estudiantes evaluados incorporan las correcciones dadas por el estudiante en proceso de FID en PEF. Por otra parte, la autoevaluación, donde comparten sus juicios valorativos con el estudiante en proceso de FID en PEF un total 103 estudiantes evaluados no lo incorpora.

\section{Discusión}

La utilización de la aplicación de evaluación formativa en procesos de FID en PEF es fundamental para mejorar y/o reorientar para beneficiar los procesos de enseñanza aprendizaje, como también lo concluye López Pastor et al., (2019) quienes plantean que la importancia de la utilización de la evaluación formativa y compartida en la formación del profesorado de Educación Física genera buenos resultados en aprendizajes de competencias docentes. Lo que a su vez concuerda con (Barrientos H, López Pastor \& Pérez-Brunicardi, 2018) quienes concluyen que la aplicación de evaluación formativa y compartida (EFyC) mejora la implicación y participación del alumnado en su propio aprendizaje favoreciendo el proceso de enseñanza aprendizaje.

En cuanto a la dimensión retroalimentación se puede apreciar que los estudiantes en proceso de FID en PEF realizan acciones que permiten y fortalecen la aplicación de la evaluación formativa en su práctica profesional, lo que concuerda con lo investigado por Gallardo-Fuentes, López-Pastor \& Carter-Thuieller (2020) quienes identifican una valoración positiva a la evaluación formativa y compartida (EFyC) en las asignaturas de formación del profesorado.

Respecto a la dimensión elaboración e implementación, los resultados del estudio permiten tener claro que en las situaciones más relevantes de evaluación formativa, como son la autoevaluación y la coevaluación, son aplicadas en un porcentaje bajo del estudiantado en FID, durante su práctica profesional. Esto refuerza la idea que usan en forma más bien intuitiva acciones de evaluación formativa (Pasek de Pinto \& Mejía, 2017) y corresponden más a acciones definidas a partir de la metodología de enseñanza y donde consideran algunos principios pedagógicos, más que un uso consciente y sistemático de lo que es la evaluación formativa como proceso, ligado al aprendizaje y con las características que debe tener como evaluación formadora que estimule la autorregulación de los aprendizajes, como señala (Sanmartí, 2007) y la autonomía en los estudiantes. Manejan algunos aspectos recurrentes como la retroalimentación, pero otros elementos que son muy importantes en el modelo de la evaluación para el aprendizaje como la incorporación de la información para generar procesos reflexivos de autoevaluación, de aplicación de coevaluación, no se evidencian en sus intervenciones en las clases observadas. Por ello se hace necesario considerar el buen uso de la retroalimentación para el desarrollo de competencias superiores en los estudiantes en FID (Asún et al., 2020).

Que no se apliquen de manera frecuente la auto y coevaluación en los procedimientos aplicados por los y las profesores/as de FID en PEF, priva a los estudiantes de la posibilidad de desarrollar autonomía, autoaprendizaje y capacidades de metacognición, al carecer de espacios para darse cuenta de sus errores y adquirir conciencia de cómo superarlos y qué hacer para mejorar (Ramírez, 2011).

En la dimensión respuesta de los escolares queda en evidencia que los estudiantes incorporan la información otorgada como retroalimentación por los estudiantes en proceso de FID en PEF durante la aplicación de la evaluación formativa en el desarrollo de su práctica profesional, pero este no propicia espacios de participación de los escolares en los procesos de evaluación, ni se establece en conjunto con ellos acuerdos referidos a criterios, ni se los involucra en procesos de autoevaluación y evaluación entre pares en relación a los resultados presentados. Si consideramos que la mejora del aprendizaje según (Broadfoot, et al., 1999) depende de cinco factores claves: la provisión de retroalimentación efectiva a los alumnos, el involucramiento activo de los alumnos en su propio aprendizaje, el ajuste de la enseñanza te- 
niendo en cuenta los resultados de la evaluación, un reconocimiento de la profunda influencia que tiene la evaluación sobre la motivación y la autoestima de los escolares, se aprecia en la presente investigación que no todos estos factores son considerados. Además, al no propiciar la autoevaluación se afecta el desarrollo de la metacognición, y darse cuenta de sus fortalezas y debilidades en el aprendizaje, lo que se relaciona con lo investigado por Ravela, Picaroni y Loureiro (2017).

Algo preocupante en los resultados es que un porcentaje disminuido (6 o menos indicadores) de uso de acciones que muestran el manejo de procedimientos de evaluación formativa, concordando con Garcia-Medina (2020) que existe una necesidad de promover la mejora de las prácticas de evaluación formativa de los y las docentes.

Se puede ver en relación a los resultados presentados en el este artículo que los estudiantes en proceso de FID en PEF que aplican evaluación formativa en el desarrollo de su práctica profesional lo hacen de manera positiva, sin descalificaciones, dando indicaciones precisas y claras, otra acción que se observa es que estimulan a los que tienen mayores problemas para lograr los aprendizajes y que hacen preguntas para que aquellos que presentan errores puedan darse cuenta de lo que les está causando la complicación. Refuerzan al cierre de la clase y señalan tareas para que practiquen y superen dificultades. Esto concuerda con lo investigado por Gallardo-Fuentes \& Carter Thuillier (2015), quienes señalan que la aplicación no evidencia desventajas y se da una interacción constante mediante el feedback y que se valora la experiencia. La aplicación de la evaluación formativa traerá mejoras en el proceso de enseñanza aprendizaje como se presenta en esta investigación, tal como señalan Hortigüela, Pérez \& Moreno (2018), en donde mencionan que la evaluación formativa representa la mejor manera de lograr resultados significativos que propicien cambios, ajustes y trasformaciones de las realidades educativas. En este mismo sentido, (Hernández \& Salicetti, 2018), señalan que la evaluación formativa favorece la interrelación profesor - estudiante, estimulando la comprensión de lo aprendido, la reflexión e implementación de los aprendizajes alcanzados.

\section{Conclusión}

Se evidencia que los estudiantes en proceso de FID en PEF realizan acciones en que aplican la evaluación formativa en su práctica profesional, especialmente re- troalimentación.

Otra conclusión que se plantea es que elementos importantes en el modelo de la evaluación para el aprendizaje como generar procesos reflexivos de autoevaluación y la aplicación de coevaluación, no se evidencian en las intervenciones en las clases observadas.

En relación a determinar las respuestas de los escolares frente a la evaluación formativa implementada por los estudiantes en FID en su práctica profesional, es posible concluir que los escolares incluyen las correcciones que los estudiantes en FID les realizan, sin embargo, se evidencia que los estudiantes en FID no propician espacios de diálogos que generen participación y el involucrarse de los escolares, respecto al proceso evaluativo.

Si bien es un estudio que considera una cohorte de estudiantes, de una carrera, en una sola institución, la información aportada permite tener claro qué es lo logrado en la formación por el grupo estudiado y confirmar la necesidad de fortalecer los procesos formativos de los estudiantes de la carrera de pedagogía en Educación Física en temas sensibles como el abordado en este caso, aplicación de evaluación formativa, que es un problema detectado en la formación de profesores.

Los resultados del presente estudio permitirán orientar en forma más clara y fundamentada lo que se debe realizar en los procesos formativos de la carrera para fortalecer lo que se detecta como positivo en el desempeño de los estudiantes en su práctica final y, además, definir estrategias que permitan superar las deficiencias detectadas en este estudio. ¿Por qué se mantiene esta situación si las normativas legales apoyan y estimulan la aplicación de evaluación formativa en los ámbitos de enseñanza aprendizaje de la escuela y por lo tanto es una competencia que deben evidenciar los futuros profesores/as? ¿Los docentes formadores de profesores/as para el sistema escolar propician el modelo de evaluación formativa en sus clases? ¿Se logran experiencias que permiten al estudiante en FID vivenciar las ventajas de una evaluación que apoye los aprendizajes? Son interrogantes que direccionan los esfuerzos investigativos siguientes.

\section{Referencias}

Agencia de la calidad de la Educación. (2016). Estudio sobre formación inicial docente en evaluación educacional.

Álvarez Méndez, J. (1993). Evaluar para conocer, examinar 
para excluir. Ediciones Morata S.L. Madrid.

Asún S, Fraile A, Aparicio, JL, Romero Ma (2020). Dificultades en el uso del feedback en la formación del profesorado de Educación Física. Retos, 37(37), 85-92. https: / /doi.org/10.47197/retos.v37i37.71029

Barrientos Hernán, E., López Pastor, V., \& PérezBrunicardi, D. (2018). ¿Por qué hago evaluación formativa y compartida y/o evaluación para el aprendizaje en EF? La influencia de la formación inicial y permanente del profesorado. Retos, 36(36), 37-43. https://doi.org/ $10.47197 /$ retos.v36i36.66478

Black, Paul \& D. Wiliam (2009). "Developing a theory of formative assessment». Educational Assessment, Evaluation and Accountability. 21, (1): 5-31.

Broadfoot, P. M., Daugherty, R., Gardner, J., Gipps, C. V., Harlen, W., James, M., et al. (1999). Assessment for learning: Beyond the black box. Cambridge, UK: University of Cambridge School of Education.

DinanThompson, M., \& Penney, D. (2015). Assessment literacy in Primary Physical Education. European Physical Education Review, 21 (4), 485-503. https: / /doi.org/10.1177/1356336X15584087

Gallardo-Fuentes, F., \& Carter Thuillier, B. (2015). La evaluación formativa y compartida durante el prácticum en la formación inicial del profesorado:Análisis de un caso en Chile. Retos, (29), 258-263. https://doi.org/ $10.47197 /$ retos.v0i29.43550

Gallardo-Fuentes, F., López-Pastor, V., \& CarterThuillier, B. (2020). Ventajas e Inconvenientes de la Evaluación Formativa, y su Influencia en la Autopercepción de Competencias en alumnado de Formación Inicial del Profesorado en Educación Física. Retos, 38(38), 417-424. https: / /doi.org/10.47197/retos.v38i38.75540

Hamodi, C. López Pastor, V. \& López Pastor, A. (2015). Medios, técnicas e instrumentos de evaluación formativa y compartida del aprendizaje en educación superior. Perfiles educativos. 37(147), 146-161. https:// www.redalyc.org/articulo.oa?id=132/ 13233749009

López Pastor, V., Molina Soria, M., Pascual Arias, C., \& Manrique Arribas, J. (2019). La importancia de utilizar la Evaluación Formativa y Compartida en la Formación Inicial del Profesorado de Educación Física: los Proyectos de Aprendizaje Tutorado como ejemplo de buena práctica. Retos, 37(37), 620-627. https://doi.org/10.47197/ retos.v37i37.74193

López Pastor, V., Molina, M., Pascual C., \& Manrique, J. (2020). La importancia de utilizar la Evaluación Formativa y Compartida en la Formación Inicial del Profesorado de Educación Física: los Proyectos de Aprendizaje Tutorado como ejemplo de buena práctica. Retos, N³7. 620 -627.

López, C., Benedito, V., \& León, M. (2016). El enfoque de competencias en la formación universitaria y su impacto en la evaluación: la perspectiva de un grupo de profesionales expertos en Pedagogía. Formación universitaria, 9(4), 11-22. doi: 10.4067/S071850062016000400003

López-Pastor V. M. \& Pérez-Pueyo, A. (coords.) (2017). Evaluación formativa y compartida en Educación: experiencias de éxito en todas las etapas educativas. León: Universidad de León. http:// buleria.unileon.es/handle/10612/5999

López-Pastor, V. M. (coord.) (2009). La Evaluación Formativa y Compartida en Educación Superior: propuestas, técnicas, instrumentos y experiencias. Narcea: Madrid.

Moreno, T. (2012). La evaluación de competencias en educación. Revista Sinéctica, 2012, Vol,39.01-20.http:// w w w. s c i e l o . o r g. $\mathrm{m}$ x / scielo.php?script $=$ sci_arttext\&pid $=$ S 1665 109X2012000200010\&lng=es\&nrm=iso

Pedro Ravela, B., \& Loureiro, G. (2017) ¿Cómo mejorar la evaluación en el aula? Grupo Magro Editores, México.

Ramírez Navarro, M. (2011) Evaluar contextos para entender el proceso del aprendizaje, cap. 5. La Evaluación Formativa. Jalisco. México.

Stobart, G. (2006) Tiempo de prueba: Ed Morata. Madrid. España.

Wiliam, D., \& Thompson, M. (2007) Integrating assessment with instruction: What will it take to make it work? In C. A. Dwyer (Ed.), The future of assessment: Shaping teaching and learning. Mahwah, NJ: Lawrence Erlbaum Associates.

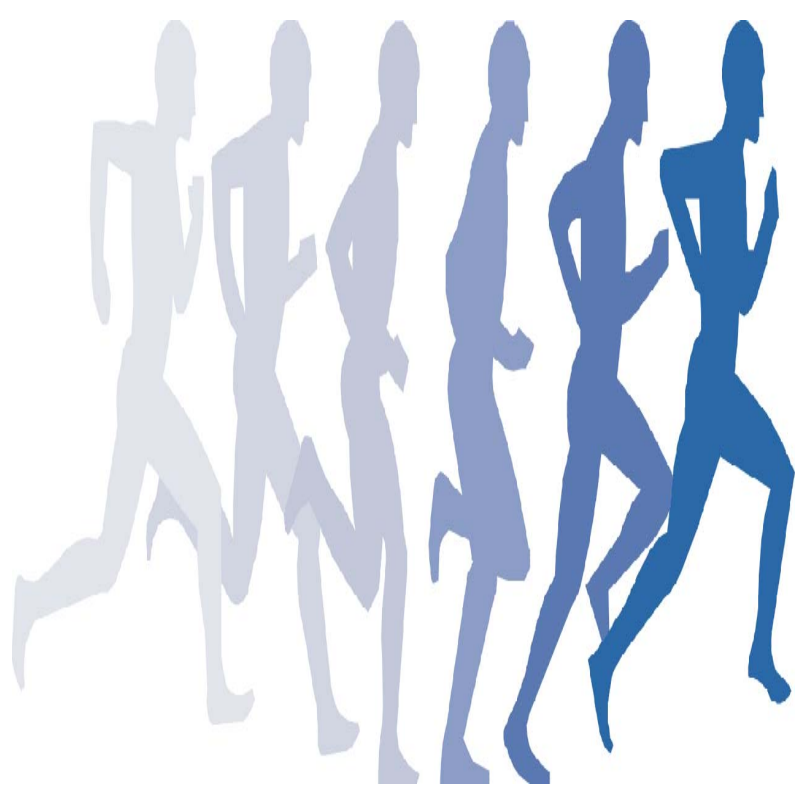

